\title{
Influencia de los patrones de uso actual de la tierra sobre la dinámica de la vegetación en el páramo de Rabanal, vereda San Antonio, municipio de Guachetá
}

\author{
Luis Miguel Borrás S., Guillermo Alfredo Galindo S. \& Wilson Triviño Gíl \\ lumibo30@hotmail.com, guillerags@hotmail.com,wtriviño@hotmail.com \\ Universidad Nacional Abierta y a Distancia (UNAD) \\ Escuela de Ciencias Agrícolas, Pecuarias y de Medio Ambiente (ECAPMA) \\ CEAD Tunja, Colombia
}

Resumen.- Los patrones del uso actual de la tierra en el páramo de Rabanal parecen indicar un lento y progresivo deterioro de la vegetación característica de este ecosistema, debido probablemente a su régimen actual de disturbios impuesto al parecer en gran medida por causas humanas, evidenciándose principalmente la ampliación de la frontera agrícola (cultivos de papa mecanizados) y pastoreo. La situación característica en la cual se llevan a cabo los patrones de ocupación y tenencia de la tierra en estos lugares, así como el sistema de producción que se establece son el punto de partida para determinar su efecto sobre este sector del ecosistema paramuno de Rabanal y definir si realmente esta circunstancia es determinante para buscar su conservación y manejo.

El trabajo se realizó en tres etapas que consistieron en la caracterización de la zona, la cual se realiza bajo la recolección de encuestas, revisión de información primaria y secundaria, seguida de un trabajo de campo, para finalizar en la tabulación de la información.

El presente estudio arroja unos resultados que pretenden aportar al conocimiento de uso actual de la tierra en un sector de la vereda San Antonio - municipio de Guachetá, inmediato al páramo de Rabanal y con este dar las bases necesarias para comprender la forma como opera la intervención antropogénica en el área y sus implicaciones en la dinámica de la vegetación, y el conocimiento de sus sistemas de producción.

Palabras clave: Antropogénica, Disturbio, Páramo, Uso de la Tierra

Abstract.- The patterns of current land use in the Rabanal paramo suggest a slow and progressive deterioration of the vegetation of this ecosystem, probably due to the current practice of disturbances imposed apparently largely by human causes, mainly the expansion of the agricultural frontier (mechanized potato crops) and grazing. The typical situation of the pattern of occupation and land-holding in these places, as well as the established production system, is the starting point to determine its effect on this sector of the Rabanal paramo ecosystem and define whether or not this fact is crucial to its conservation and management.

The study was conducted in three stages that included the characterization of the area done through surveys, a review of primary and secondary education, followed by field work, and final tabulation of information.

The present study obtained results which were intended to contribute to the knowledge of current land use in a sector of the settlement of San Antonio - Township Guachetá, bordering the Rabanal Paramo and with this give the necessary foundation for understanding how anthropogenic intervention operates in the area and its implications for vegetation dynamics and knowledge of its production systems.

Keywords: Anthropogenic, disturbance, Paramo, Land Usage 


\section{Introducción}

Los páramos en las altas cumbres de los Andes ecuatoriales húmedos de Colombia pertenecen al ecosistema montano alto, que a su vez se integra al bioma montano. En el orden bioclimático el ecosistema montano alto presenta condiciones ambientales extremas y con grande influencia biológica (Guhl 1995). El comportamiento del hombre desde sus arraigos culturales muestra una clara tendencia a la ocupación progresiva de los ecosistemas de alta montaña, la implementación de dichos sistemas de producción en estos ecosistemas genera menoscabo de una singular muestra de recursos biológicos allí presentes, además de su probable pérdida en la capacidad de ofertar bienes y servicios ambientales a las comunidades que de allí dependen. $\mathrm{Al}$ recorrer algunos de los páramos de nuestro país se tiene la impresión de que la gran mayoría de ellos recibe poca o ninguna atención para su protección y uso racional.

Según la dinámica de uso actual del suelo en la región de la Vereda San Antonio del Municipio de Guachetá (Cundinamarca), se observa un alto grado de intervención de la zona de páramo ocasionada por la gran expansión de la frontera agrícola, lo cual ocasiona diferentes disturbios antrópicos y por consiguiente la alteración de la vegetación. La intervención del ecosistema genera la degradación del suelo y provoca la desaparición de especies naturales importantes que han caracterizado el territorio, es importante anotar que no se tiene referente de estudios similares en la zona, sin embargo Vargas \& Rivera (1990) ha llamado la atención sobre la necesidad de investigar sobre los sistemas de producción y su efecto en el medio ambiente de páramo. Este autor agrega que dicha urgencia -debido muy seguramente a la amenaza actual en que se encuentran- ha sido enfatizada recientemente en una serie de publicaciones, tales como para los páramos de Ecuador Hess (1990) y Lagaard (1992); para los páramos de Venezuela Montilla et al. (1992) y para los páramos de Colombia Schmidt \& Verweij (1992).
Los sistemas de producción que se han venido estableciendo a partir del período de la conquista española en nuestros ecosistemas paramunos, no parecen ofrecer condiciones de sustentabilidad ecológica. El uso del fuego ligado a los sistemas de producción agrícola es una práctica que se da con mucha frecuencia; de la misma manera el pastoreo de ganado vacuno, ovino, caprino y equino es otro uso tradicional practicado en las unidades agrícolas familiares de áreas de minifundio con el fin de mejorar el nivel de ingresos, que en la mayoría de las ocasiones es bajo.

El presente estudio sobre la influencia de los patrones de uso actual de la tierra sobre la dinámica de la vegetación "vereda San Antonio, municipio de Guachetá, departamento de Cundinamarca”, presenta una caracterización sobre el uso actual de la región, determina los tipos de conflictos sobre uso y manejo del suelo, y plantea soluciones al respecto tomando como base cuatro unidades de producción. Se puede presumir que la dinámica espacial y el funcionamiento de los patrones de arreglo de la vegetación y los elementos del paisaje en este ecosistema de páramo se están viendo modificados por el uso actual de la tierra.

Para Vargas \& Rivera (1990) señalan los siguientes factores que están influyendo aceleradamente en la destrucción de los páramos en Colombia.

- Quemas indiscriminadas

- Ganadería extensiva

- Erosión hídrica fuerte y alteración de los movimientos naturales del agua

- Sedimentación y eutrofización de las lagunas

- Corte de matorrales y bosques enanos para leña

- Extinción de fauna y flora nativas. Pérdida de endemismos

- Desviación de las sucesiones naturales

- Pérdida del potencial de regeneración natural 
- Explotación de minas

- Colonización acelerada

- Establecimiento de plantaciones forestales con especies exóticas (Cupresus sp., Pinus sp.).

- Apertura de carreteras

La región objeto de estudio perteneciente a la parte occidental del páramo de Rabanal. Presenta en su gran mayoría las manifestaciones descritas anteriormente, con la consecuente degradación de los recursos y la alteración de las características estructurales y funcionales de los ecosistemas allí presentes. Es muy importante observar de cerca los impactos de estos disturbios sobre todo los ocasionados por las actividades socioeconómicas del sector con el fin de analizar su desenvolvimiento bajo el régimen actual y de esta manera contribuir al conocimiento como base para determinar la necesidad de su adecuado manejo.

Esto finalmente permite establecer el régimen de disturbios típico del área y su grado de intervención sobre la vegetación que en parches aislados aún subsiste.

\section{Objetivo General}

Evaluar los patrones de uso de la tierra en inmediaciones del páramo de Rabanal, vereda San Antonio y su influencia en la dinámica de la vegetación.

\section{Objetivos Especificos}

- Evaluar cuatro unidades de producción para determinar el sistema de producción local

- Caracterizar los patrones de sucesión - regeneración que generan los sistemas de producción en la zona de estudio identificada, en inmediaciones del páramo de Rabanal, vereda San Antonio del municipio de Guachetá

- Analizar los patrones de uso de la tierra como base del conocimiento de la dinámica de la vegetación impuesta por las unidades de producción

\section{Materiales y métodos}

El tipo de estudio se enmarca dentro de los parámetros analítico descriptivos en el desarrollo del estudio se realizaron los siguientes pasos:

\section{Diagnóstico}

El estudio se llevó a cabo paralelamente con un trabajo de fotointerpretación y cartografía, con ayuda de información de tipo primario y secundario obtenida a partir de la recopilación bibliográfica y cartográfica como de visitas de campo.

Los estudios de finca para construir modelos cualitativos y cuantitativos, generalmente se hace por medio de encuestas y registros de fincas. Trabajar con modelos de finca ayuda a entender cómo funciona el sistema y la posibilidad de introducir modificaciones dentro de un ambiente más predecible que una finca real.

\section{Reconocimiento de la Región}

Se realizó a partir del análisis de la cartografía disponible de la zona y temática básica, para analizar a un nivel general pero comprensivo, el uso actual del suelo y la cobertura vegetal, el grado de deterioro del suelo y la vegetación, las características fisiográficas y sus potencialidades de aprovechamiento, mediante una matriz que contiene las características físicas propias del municipio como hidrología, geología y clima.

\section{Reconocimiento de la Zona de Estudio}

Se practicaron diez visitas de reconocimiento de la zona donde se hizo un levantamiento del paisaje cultivado elegido, dentro del cual se escogieron cuatro unidades agrícolas familiares al azar, con sus diferentes parcelas, algunas con los cultivos propios del área, otras tienen diferentes estados sucesionales de acuerdo al patrón de producción y las últimas con muestras 
de la vegetación original. Para la elección de estas unidades se tuvieron en cuenta aspectos como el tamaño de cada unidad, intensidad en el uso del suelo, los relictos de vegetación presentes y la tenencia de la tierra.

Durante estas visitas se obtuvo la información primaria de las unidades a través de una encuesta para un modelo de finca que involucra variables socioeconómicas y tecnológicas.

Para la dinámica de la vegetación en términos de los estadios de la sucesión - regeneración en cada una de las parcelas se apoyó en los registros fotográficos de las especies indicadoras predominantes, que se presentaron en las diferentes parcelas en función del sistema de producción local. Así mismo se realizaron visitas periódicas para determinar el uso actual del terreno, observación directa por parte de los autores y la interacción permanente con los habitantes de la zona. Dentro de las actividades desarrolladas por parte de los autores del estudio, además de charlas y consultas con los habitantes de la zona de estudio, se realizó la recolección de especies vegetales propias del terreno. Por otra parte, el estudio se apoyó en información secundaria de diferentes estudios de investigación a cerca de la vegetación de páramo y la incidencia de la dinámica de uso del suelo sobre la vegetación.

\section{Análisis de la información}

Se englobaron los elementos esenciales del análisis espacial, el régimen de disturbios típico de acuerdo al patrón de usos del suelo y el estado de sucesión - regeneración para evidenciar en cada una de las parcelas el grado y escala de la intervención antropogénica. Así mismo, haciendo uso del análisis de las unidades de producción se determinó la operación dentro del enfoque de la teoría de sistemas.

La tabulación de la encuesta, se realizó mediante el uso de tablas analizando cada una de las respuestas obtenidas para llegar a conclusiones generales del tema. La metodología empleada sugiere la confrontación y análisis de la información secundaria y la literatura, el conocimiento empírico de los habitantes y las observaciones de los autores. Este análisis permitió conocer la dinámica de uso actual del suelo con base en el estado de la vegetación y los sistemas de producción imperantes, el grado de intervención al ecosistema y la dinámica de interacción del hombre con el páramo.

\section{Resultados}

\section{Características Socioeconómicas}

Uso actual del territorio

El uso de la tierra está dado por pastoreo alternada con agricultura, esta última con muy poca rotación de cultivos. En las unidades estudiadas se observó que la ocupación del área total de los predios en agricultura y pastoreo no supera en ningún caso el $40 \%$ el cual el $15 \%$ es utilizado en cultivos y el $25 \%$ restante en pastoreo. La zona tiene un predominio de pastos con parches geométricos de cultivos de papa, trigo, cebada y huertas caseras de hortalizas; también se encuentran parcelas en descanso en diferentes etapas de sucesión - regeneración, y finalmente muy pequeñas áreas de relictos de bosque natural secundario y vegetación de páramo.

El uso agrícola de la zona está representado primordialmente al cultivo de papa, cuyo período vegetativo es mayor debido a las condiciones climáticas y edáficas. Son cultivos transitorios, establecidos con técnicas inapropiadas.

El sistema de producción consiste en la implementación de dos cosechas de papa, seguido de uno de trigo o cebada y posteriormente se deja para pastoreo por un periodo aproximado de 2 años; en ocasiones se dejan parcelas en descanso por mucho más tiempo, de acuerdo a la disponibilidad de tierras del agricultor (Fig. 1).

En ocasiones se observa la implementación de cultivos transitorios mixtos e intercalados tales como avena y trigo después del cultivo de la papa. Así mismo existen zonas relictuales de ve- 


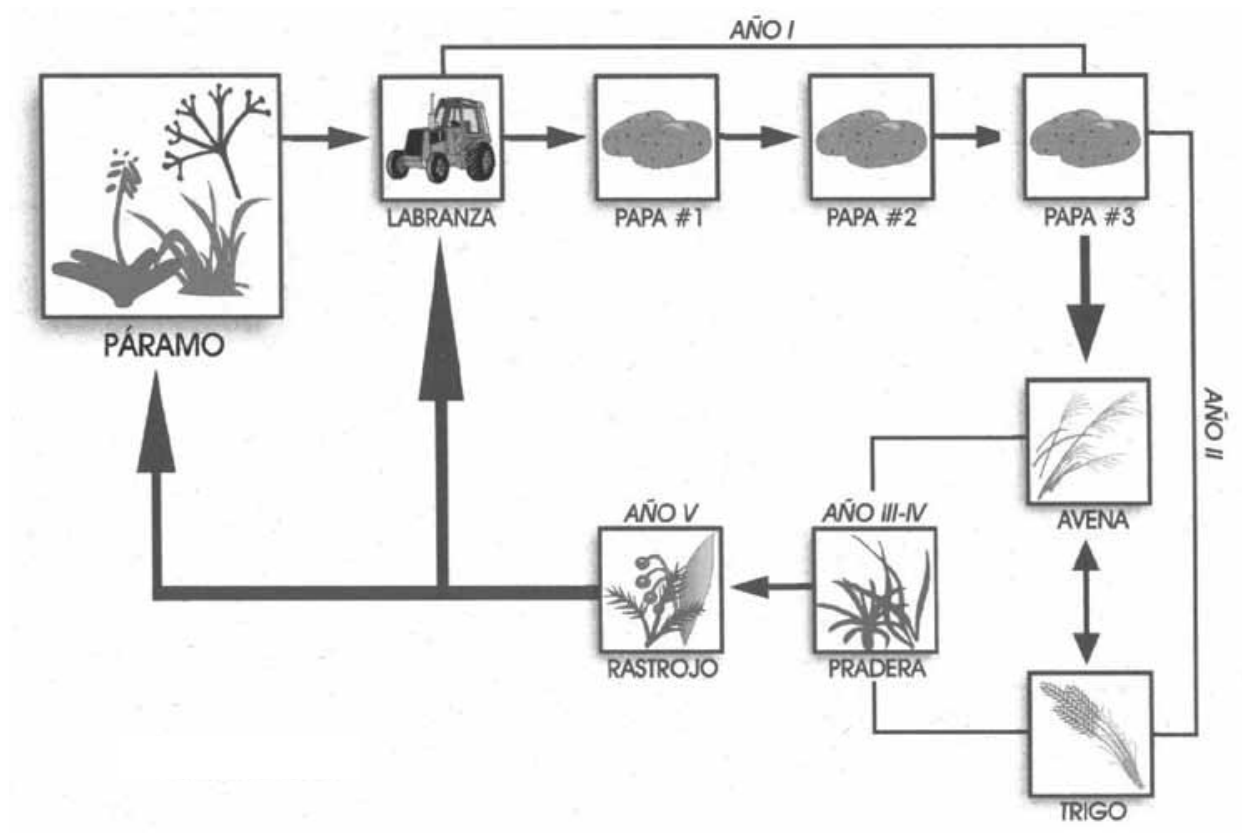

Figura 1. Esquema del sistema de producción "arriendo".

getación natural original que no son roturadas debido a la presencia de rocas y también debido a la pendiente más pronunciada (Fig. 2).

\section{Tierras en pastos}

Se encuentran lotes de pastos que prevalecen sobre el uso agrícola, estas áreas de pastoreo se encuentran en algunos predios para la críaen forma extensiva de ganado vacuno y caballar.

Pennisetum clandestinum Hochst. ex Chiov. (kikuyo) se presenta en las cotas más bajas y Rumex acetocella L., Geranium sp. y Digitalis sp. en alturas mayores.

\section{Pastos con rastrojo}

Se encuentran en áreas donde se ha disminuido la actividad pecuaria y ha comenzado el proceso sucesional con especies como Hypericum sp., Espeletia argentea Bonpl., entre otras.

\section{Rastrojo}

Comprende el bosque natural secundario pionero, la unidad está representada por vegetación de tipo arbustivo.

\section{Características del agricultor y su familia} Educación

De las unidades de producción estudiadas existe un promedio de seis habitantes por vivienda, los cuales tienen un grado de escolaridad no superior al quinto de primaria, existe una escuela veredal, la cual se encuentra a $2 \mathrm{~km}$ aproximadamente de la zona de estudio.

\section{Salud}

El centro de salud se localiza en el casco urbano del municipio de Guachetá, aproximadamente a $4 \mathrm{~km}$; el principal medio de transporte utilizado es el caballo.

\section{Comunicaciones}

El único medio de comunicación posible en la vereda está dado por un radio - teléfono, el cual se ubica en la escuela.

\section{Combustible utilizado}

La vereda se encuentra casi en su totalidad con servicio de electricidad, sin embargo el combustible más utilizado es el carbón mineral, máxime cuando este municipio es de gran influencia minera. 


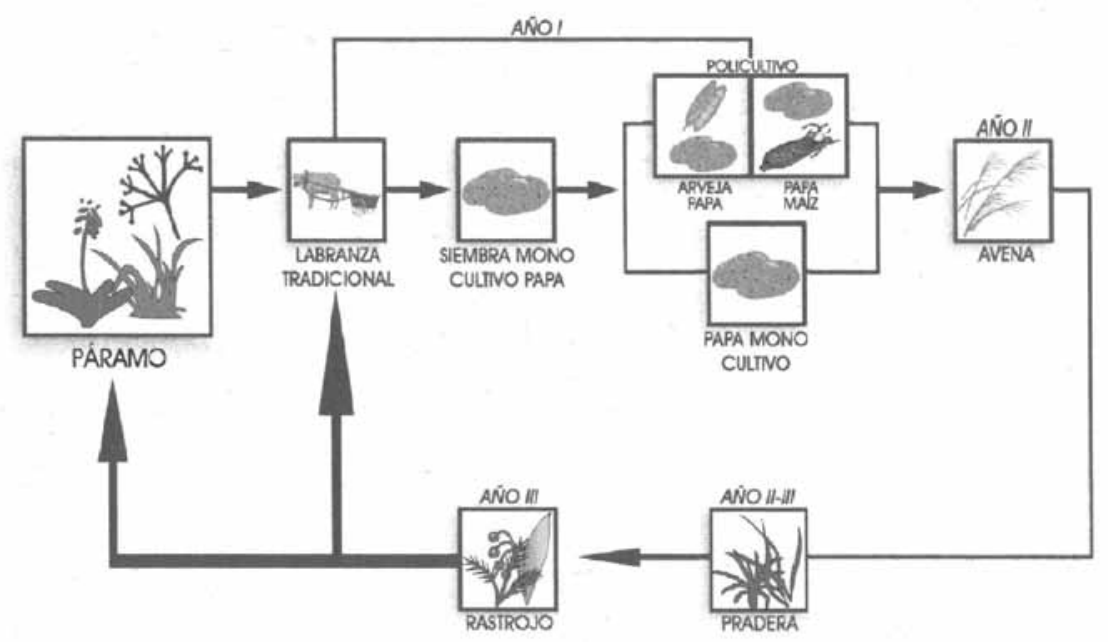

Figura 2. Esquema del sistema de producción "propietarios".

\section{Asistencia técnica}

Las personas encuestadas manifestaron que la única asistencia técnica a la cual tienen acceso es la suministrada por el proyecto Checua, la cual es totalmente gratuita, además manifiestan que el acceso a los créditos no es muy factible, y en el caso de llegar a acceder a uno de ellos no se les suministra ningún tipo de asistencia técnica.

\section{Tecnología de producción}

La tecnología empleada en los sistemas de producción de las dos unidades de propiedad de los pobladores, es rudimentaria, se aprecia la utilización de la fuerza animal representada en caballos, asnos y bueyes. Se utilizan elementos de labranza mínima como el arado de madera y el azadón. En las dos unidades en arriendo predomina la agricultura semimecanizada, con la utilización de tractor y arado mecánico.

\section{Principales productos}

Los productos obtenidos de las cosechas son comercializados en un $70 \%$. Los cuales se distribuyen en los mercados de Guachetá y Ubaté, y los productos restantes son consumidos en su alimentación como los obtenidos en la huerta casera (maíz y habas).

\section{Insumos utilizados}

En el cultivo de la papa se utilizan principalmente fertilizantes, abonos orgánicos como equinaza y otros. Dentro de losabonos químicos el 15-15-15, herbicidas y fungicidas empleados tradicionalmente.

\section{Especies pecuarias}

Las principales especies que predominan en estas zonas son los bovinos, especialmente razas de doble propósito como es el normando, el cual les proporciona leche principalmente para consumo y algunos excedentes para la venta. También se crían ovinos y algunos caprinos que generalmente se comercializan en el mercado regional. Los equinos son utilizados básicamente para carga y transporte. Se crían algunas especies menores tales como conejos y gallinas las cuales hacen parte de la dieta básica. Estos sistemas de producción tienen como característica que la cría y desarrollo se realiza en forma extensiva sin ningún tipo de asesoría.

Características Bióticas

Vegetación

Existen algunos lugares pequeños aislados de vegetación original conformados en algunos 
casos por especies de tipo arbustivo entre pajonales y frailejonales (Espeletia sp., Paspalum hirsutum Poir. y Anthoxantum odoratum L.) que hacen presumir que se trata de vegetación transicional entre el bosque altoandino y el subpáramo.

De la misma manera existen en las diferentes parcelas vegetación en diferente grado de sucesión que se discute más adelante, de acuerdo a su periodo de descanso.

Factores determinantes en las sucesiones impuestas por los sistemas de producción

- El periodo de descanso: estrategia de conservación y diversidad. También como regeneración de la fertilidad

- El banco natural de semillas: reserva de semillas que determina el potencial de regeneración natural (en los dos primeros centímetros se encuentra la mayor densidad y diversidad de semillas viables)

- Régimen de disturbios: la falta de prácticas de conservación perturban más al ecosistema, y desvían y detienen las sucesiones

\section{Sucesiones encontradas y sus fases}

Parcelas en varias fases de sucesiones secundarias:

FASE 1: sucesiones luego de los primeros años de descanso de las parcelas; especies oportunistas de gramíneas introducidas (desde el $1^{\circ}$ año de descanso hasta el $3^{\circ}$ o $4^{\circ}$ año) si hay pastoreo las sucesiones se detienen

FASE 2: aparecen especies colonizadoras del páramo (entre el $4^{\circ}$ y $7^{\circ}$ año)

FASE 3: especies de arbustos: Vaccinium sp. y otros (entre $7^{\circ}$ y $10^{\circ}$ año)

FASE 4: vegetación clímax: gramíneas fasciculadas de géneros: Calamagrostis sp. y Festuca sp. y las formas caulirrosulas de Espeletia sp. Para que los frailejones lleguen de nuevo a alturas de 2 m pueden transcurrir entre 50 y 100 años. En general la vegetación se encuentra en todas las parcelas bajo fuerte influencia humana y en estado secundario constante.

\section{Discusión y conclusiones}

\section{Análisis de las unidades de producción desde un punto de vista sistémico}

Una forma sencilla de entender la dinámica de las unidades de producción estudiadas es verlas desde un punto de vista sistémico, lo cual nos facilita la interpretación de los componentes y flujos de los agroecosistemas allí establecidos.

El modelo del sistema está compartido por cuatro subsistemas bien definidos; el principal definido como subsistema de cultivos, seguido por el subsistema de praderas; estos dos interactuando muy de cerca con el subsistema pecuario, encontrando uno muy importante pero a su vez el más deprimido y del cual se generan los otros tres, el subsistema natural o de rastrojo natural. El subsistema rastrojo natural guarda un registro de lo que fue el ecosistema en su estado natural y ha servido de parámetro de cotejo para analizar y entender toda la dinámica poblacional y las sucesiones que allí se han generado con todos y cada uno de los disturbios allí realizados por la influencia antrópica en la zona.

\section{Sistemas de producción y sus implicaciones ecológicas con especial referencia a la dinámi- ca sucesional de la vegetación}

Como se mostró en los resultados se hallaron dos sistemas de producción, los cuales a su vez corresponden a un sistema mayor que podría denominarse de alta montaña en ladera (2.800$3.200 \mathrm{msnm}$ ) en condiciones de clima frío a subpáramo.

Los dos sistemas de producción hallados están determinados esencialmente por el uso de la tierra, es decir, si el objetivo es producir para autoconsumo con algunos excedentes para comercialización, o solamente para venta.

Obviamente las implicaciones en ambos casos son muy diferentes dependiendo del funcionamiento del sistema, en cuanto a tecnologías, in- 
gresos y salidas de energía, grado de intervención, sobre el suelo, tamaño y número de agroecosistemas de cada finca y arreglos entre cultivos (distribución en el espacio y en el tiempo).

En el sistema de producción propietarios donde el objetivo es la subsistencia, opera un mayor control analítico sobre las parcelas, es decir, se planifica de alguna manera los arreglos en los cultivos atendiendo al estado de cada una de estas y su actividad anterior.

Cabría de esperarse que los subsidios energéticos que se introducen al sistema estuvieran en función de la actividad anterior del suelo, con el avance del estado sucesional y la presencia de nutrientes en forma de fitomasa pero el uso de fertilizantes químicos es una constante que muestra una pérdida de las prácticas tradicionales ancestrales. Aunque se utilizan fertilizantes, esto no quiere decir que no se tenga en cuenta la fertilidad de las parcelas a ser cultivadas en términos de biomasa a ser reincorporada en el periodo de arado o barbechado, el cual es el punto de partida de este sistema de producción; teniendo en cuenta también otros aspectos como la estación climática, la disponibilidad de mano de obra por parte de la unidad familiar y también sus condiciones económicas.

En este sistema de producción: propietario, se presenta una mayor diversidad en términos de parcelas o agroecosistemas debido a la reducida producción, que en el sistema de arriendo, por tanto el tamaño de las parcelas a ser cultivadas es menor, lo cual se refleja en un mosaico más variado de parcelas en diferentes estados.

De acuerdo a lo anterior se puede precisar que los atributos de los diferentes agroecosistemas dentro de cada finca, se reflejan en términos de fitoindicadores, los cuales varían en función de su utilización, es decir los diferentes arreglos de cultivos y la presencia de periodos largos o cortos de descanso del suelo.
Entre mayor sea el periodo de descanso de una parcela se tiene una mayor regeneración de la fertilidad, ya que hay una mayor cantidad de biomasa, la cual es una fuente de abono, que se incorpora cuando la parcela es arada, luego de un periodo de descanso lo suficientemente prolongado para que haya una buena densidad de vegetación; la mezcla de fitomasa con la capa de tierra removida, enriquece la parcela en nutrientes luego de la descomposición y mineralización de la materia orgánica. Como punto importante cabe destacar que a diferencia de las prácticas tradicionales en otros páramos, el uso del fuego no está asociado a este sistema de producción.

Pasando a hacer un análisis del otro sistema de producción, es decir en el cual se utiliza la tierra en arriendo, ya existen unas mayores inversiones y por lo tanto unos mayores subsidios a los agroecosistemas; en la preparación del terreno ya hay una dependencia de la mecanización, existe fertilización química y un mayor control fitosanitario. Se amplían las áreas de cultivo y por tanto, una mayor uniformidad del paisaje. Aquí hay una menor atención a las expresiones naturales de las parcelas, como una variable prioritaria para elegir el tiempo propicio para empezar los cultivos.

Si bien existe algún tipo de patrón en los sistemas de utilización de la tierra en el sector, hay una serie de variables, más que todo del tipo socioeconómico que relativizan, por así decirlo, la clasificación que toma como partida la tenencia de la tierra, por ejemplo el sistema de producción del tipo propietario está en una alta proporción abandonando las prácticas tradicionales y optando por la agricultura de revolución verde o un poco más asía la convencional. Teniendo en cuenta la dinámica sucesión - regeneración, se pudo constatar en función de los resultados, que lo más determinante en la expresión florística de una parcela determinada se encuentre en un estado subseral determinado como el periodo de descanso o tiempo en la cual no ha sido utilizada en actividades agropecuarias; además que la composición en cuanto 
a cantidad y diversidad de especies vegetales y su arreglo en poblaciones y comunidades, refleja en alguna forma la magnitud de la intervención que produce el menoscabo de la naturaleza del suelo y la capacidad de expresión del banco natural de semillas presente en la capa superficial de este.

Según se pudo observar en los primeros estadios de la sucesión del año primero al tercero luego de haber intervenido una parcela, aparecen gramíneas que presumiblemente por su morfología y sistema radical fasciculado tienen menos dificultad en desarrollarse que las semillas de la vegetación típica aún presente en el suelo. Estas son especies introducidas como la cizaña Rumex acetosella L., el pasto de olor Anthoxanthum odoratum L. y la poa Holcus lanatus L. las cuales en su orden invaden las parcelas casi en forma homogénea durante estos primeros años de descanso, dando paso al cabo del tiempo a otras especies colonizadoras. En esta fase el sistema ecológico conformado por la parcela cultivada y en algunas ocasiones pastoreada es bastante inmaduro, la diversidad de especies es baja y el sistema presenta un grado de organización bastante simple, en el cual hay una ocupación por poblaciones de especies oportunistas que aprovechan el substrato no ocupado por ningún otro tipo de vegetación; casi se puede decir que hay una monopolización del espacio por estas especies, con una gran capacidad de germinación, en condiciones de escasa competencia.

Entre el tercer y séptimo año de descanso ya empiezan a aparecer especies pioneras como $E$. argentea, Hypericum sp., Paephalantus columbiensis Ruhland, las cuales fueron las más representativas de las parcelas observadas, haciendo cada vez más diverso y heterogéneo el agroecosistema.

En las sucesiones de las parcelas analizadas han tomado un mayor tiempo las especies pioneras del páramo, lo cual es un indicativo de que la escala espacio - tiempo durante la cual han operado los cultivos y el pastoreo de los suelos es presumiblemente mucho más amplia que las analizadas por Guhl (1995) y Van der Hammen (1998). Por cierto que desde un punto de vista histórico, esta zona ha estado bajo influencia humana durante muchos tiempo.

Es interesante anotar el papel que juegan los lugares pequeños de vegetación natural situados en proximidad a las parcelas en el proceso de sucesión - regeneración ya que operan como un mecanismo favorable de dispersión natural. En el caso nuestro, se halló un parche relictual que contenía cierto grado de complejidad y diversas especies como: Espeletiopsis corymbosa (Humb. \& Bonpl.) Cuatrec., E. grandiflora, E. argentea, Cerastium arvense L., Calamagrostis effusa (Kunth) Steud., Hypericum goyanesii Cuatrec.; es importante tener en cuenta este tipo de parches como un indicador de las tendencias de las sucesiones y su incidencia en la recuperación de las parcelas a su alrededor.

En una parcela con 10 años de reposo o descanso la diversidad de especies era considerable, las cuales aparecen espontáneamente entre praderas de gramíneas. Es de anotar que la cobertura de especies introducidas es mayor que la cobertura de especies colonizadoras del páramo, lo cual pone de manifiesto una reducción de las semillas de estas especies y un esfuerzo cada vez mayor de complejizar el sistema.

Valga anotar que dentro del patrón común de utilización de los suelos del área de estudio esta parcela de diez años de descanso es rara, ya que la mayoría de parcelas no se dejan descansar tanto tiempo, debido a un uso cada vez más intensivo, sometiendo el suelo a prácticas no sostenibles como labranzas sucesivas, cosechas continuas, suelo poco cubierto durante largos periodos de tiempo, aumento de las superficies cultivadas y con mayores subsidios de materia y energía, como es el caso impuesto por el sistema de producción de los arrendatarios, copiado cada vez con mayor fuerza en las parcelas que habían sido trabajadas bajo un esquema más ecológico. 
Si se estudia cuidadosamente el sistema de producción de papa en los páramos, se aprecia que la recuperación, regeneración o rehabilitación de la flora nativa (salvo en algunos casos que hay pérdida irreparable de especies, ya que con dichas intervenciones muchas especies propias del páramo desaparecen para siempre, como en el caso de las orquídeas) es función de acabar o atenuar ostensiblemente la intensidad y la prolongación de intervención de estos suelos, con los objetivos de preservar y conservar el sistema ecológico ya que puede tardar varios periodos de tiempo en el proceso sucesional dependiendo de los bancos de semillas viables y dispersores.

Muchos autores anotan que es bastante difícil volver a condiciones de páramo originales ya que en su construcción ha jugado un papel importante la acción humana que ha creado sus condiciones; es decir que los seres humanos, de alguna manera han incidido en la conformación de los páramos.

La necesidad de garantizar la sostenibilidad de los recursos biológicos del páramo, racionalizar su utilización, superar las secuelas impuestas por los regímenes de disturbios ocasionados por los sistemas de producción, planificar el uso del suelo, trazar estrategias que atiendan las necesidades de la población incluyendo la transformación tecnológica, la extensión real de los proyectos de investigación científica para superar el conflicto creado por la expansión de la frontera agrícola.

A lo largo del estudio se hizo evidente que los factores antropogénicos imprimen una serie de características bastante notables a las áreas de páramo. En el caso del Rabanal se pudo apreciar la forma en que el paisaje se ha transformado por los sistemas de producción agrícolas y pecuarios que han estado interactuando con las manifestaciones vegetales.

En la mayoría de los casos los páramos están modelados o condicionados estructural y fun- cionalmente por los cultivos, es necesario estudiar o realizar un análisis de las áreas críticas en donde las acciones antropogénicas impliquen la inminente desaparición de especies.

En cuanto a la economía sobresalen los altos costos de producción, la falta de asistencia técnica, el desempleo por dependencia de la producción de carbón, por la comercialización de la leche y la intermediación entre el mercadeo y la dependencia comercial de Ubaté y Bogotá.

La ampliación de la frontera agrícola en el páramo de Rabanal, genera disminución en el nivel freático (áreas de recurso de agua) y contaminación por agroquímicos, por el rompimiento y la tala de bosque nativo en la zona, causando degradación al suelo y pérdida de sus propiedades.

Teniendo en cuenta la cabida total en superficie de las unidades de producción, las unidades de producción I y II que corresponden a los propietarios está dominado en un $60 \%$ por rastrojo natural y solo un $20 \%$ en papa, y caso contrario ocurre en las unidades III y IV: arrendatarios, el cultivo de papa se incrementa a un $50 \%$ de su área total, lo cual refleja una mayor área en sucesiones vegetales en los casos en que la intervención es menos intensa.

\section{Referencias}

Guhl, E. 1995. Los páramos circundantes de la sabana de Bogotá. FEN, Bogotá.

Hess, C.G. 1990. "Moving up-moving down”: agropastoral land use patterns in the ecuatorian paramos. Mountain Research and Development, 10(4): 333-342.

Lagaard, S. 1992. Influence of fire in the grass páramo vegetation of Ecuador. En: H. Balslev \& J.L. Luteyn (Eds.). Páramo. An andean ecosystem under human influence. Academica Press, San Diego. pp. 151-170. 
Montilla, M., R.A. Herrera \& M. Monasterio. 1992. Micorrizas vesículo - arbusculares en parcelas que se encuentran en sucesión - regeneración en los Andes Tropicales. Suelo y Planta, 2(1): 59-70.

Schmidt, A.M. \& P.A. Verwij. 1992. Forage intake and secondary production in extensive livestock systems in páramo. En: Balslev, H. \& J.H. Luteyn (Eds.). Páramo. An andean ecosysten under human influence. Academic Press, San Diego. pp. 197-210.

Van der Hammen, T. 1998. Plan ambiental de la cuenca alta del río Bogotá. Análisis y orientaciones para el ordenamiento territorial. CAR, Bogotá.

Vargas R., O. \& D. Rivera. 1990. El Páramo un ecosistema frágil. Cuadernos de Agroindustria y Economía Rural. Universidad Javeriana, Bogotá.

Recibido: 27 de septiembre de 2010 Aceptado: 15 de diciembre de 2010 